\title{
A Characterization on Singular Value Inequalities of Matrices
}

\author{
Wei Dai $(\mathbb{D}$ and Yongsheng Ye $\mathbb{C}$ \\ School of Mathematical Sciences, Huaibei Normal University, Huaibei 235000, China \\ Correspondence should be addressed to Yongsheng Ye; yeysh66@163.com \\ Received 4 December 2019; Accepted 30 December 2019; Published 18 February 2020 \\ Academic Editor: Yuri Latushkin
}

Copyright (c) 2020 Wei Dai and Yongsheng Ye. This is an open access article distributed under the Creative Commons Attribution License, which permits unrestricted use, distribution, and reproduction in any medium, provided the original work is properly cited.

We obtain a characterization of pair matrices $A$ and $B$ of order $n$ such that $s_{j}(A) \leq s_{j}(B), j=1, \ldots, n$, where $s_{j}(X)$ denotes the $j$-th largest singular values of $X$. It can imply not only some well-known singular value inequalities for sums and direct sums of matrices but also Zhan's result related to singular values of differences of positive semidefinite matrices. In addition, some related and new inequalities are also obtained.

\section{Introduction}

Let $M_{n}$ denote the vector space of all complex $n \times n$ matrices, and let $H_{n}$ be the set of all Hermitian matrices of order $n$. We always denote the eigenvalues of $A \in H_{n}$ in decreasing order by $\lambda_{1}(A) \geq \lambda_{2}(A) \geq \cdots \geq \lambda_{n}(A)$. For $A, B \in H_{n}$, we use the notation $A \leq B$ or $B \geq A$ to mean that $B-A$ is positive semidefinite. Clearly, " $\leq$ " and " $\geq$ " define two partial orders on $H_{n}$, each of which is called Löwner partial order. In particular, $B \geq O$ (res., $B>O$ ) means that $B$ is positive semidefinite (res., $B$ is positive definite). For $T \in M_{n}$, the singular values of $T$, denoted by $s_{1}(T), s_{2}(T), \ldots, s_{n}(T)$, are the eigenvalues of the positive semidefinite matrix $|T|=(T * T)^{1 / 2}$, enumerated as $s_{1}(T) \geq s_{2}(T) \geq \cdots \geq s_{n}(T)$ and repeated according to multiplicity. It follows that the singular values of a normal matrix are just the moduli of its eigenvalues. In particular, if $T \in M_{n}$ is positive semidefinite, then singular values and eigenvalues of $T$ are the same. For more information on this related topic, see [1-3]. Recall that a complex matrix $C \in M_{n}$ is called contraction if $C^{*} C \leq I$, or equivalently $\|C\| \leq 1$, where $\|\cdot\| I$ denotes the spectral norm, the identity matrix of order $n$, respectively.

Here, we denote the block matrix $\left(\begin{array}{cc}A & 0 \\ 0 & B\end{array}\right)$ by $A \oplus B$.

This paper intends to give a characterization of pair matrices $A, B \in M_{n}$ such that $s_{j}(A) \leq s_{j}(B), j=1, \ldots, n$. It can generalize some singular value inequalities for sums and direct sums of matrices due to Hirzallah and Kittaneh [4]. Using this characterization, we give a new proof of Zhan's result related to singular values of differences of positive semidefinite matrices [5]. Several applications of this characterization are presented, and some related and new inequalities are also obtained.

\section{Main Results}

The following well-known results are due to Ky Fan.

Lemma 1. Let $A, B \in M_{n}, 1<i, j \leq n$, and $i+j-1 \leq n$. Then

$$
s_{i+j-1}(A B) \leq s_{i}(A) s_{j}(B) .
$$

In particular,

$$
\begin{aligned}
& s_{j}(A B) \leq A s_{j}(B), \\
& s_{j}(A B) \leq\|B\| s_{j}(A), \\
& \quad j=1, \ldots, n,
\end{aligned}
$$

where $\|\cdot\|$ denotes the spectral norm.

Next, we will prove the following useful result.

Theorem 1. Let $A, B \in M_{n}$. Then,

$$
s_{j}(A) \leq s_{j}(B), \quad j=1,2, \ldots, n,
$$


if and only if contractive matrices $W$ and $V$ exist such that $A=W B V$. In particular, suppose $A$ and $B$ are positive semidefinite. Then,

$$
s_{j}(A) \leq s_{j}(B), \quad j=1,2, \ldots, n,
$$

if and only if there exists a contractive matrix $W$ such that $A=W B W^{*}$

Proof. The sufficiency follows from Lemma 1. We prove the necessity.

Consider the singular value decompositions $A$ and $B$. Then, unitary matrices $U_{1}, U_{2}, V_{1}$, and $V_{2}$ exist such that

$$
\begin{aligned}
& U_{1} A V_{1}=\operatorname{diag}\left(s_{1}(A), s_{2}(A), \ldots, s_{n}(A)\right), \\
& U_{2} B V_{2}=\operatorname{diag}\left(s_{1}(B), s_{2}(B), \ldots, s_{n}(B)\right) .
\end{aligned}
$$

Setting

$$
\beta_{i}= \begin{cases}1, & s_{i}(B)=0, \\ \frac{s_{i}(A)}{s_{i}(B)}, & s_{i}(B) \neq 0 .\end{cases}
$$

Denote $W_{0}=\operatorname{diag}\left(\sqrt{\beta_{1}}, \sqrt{\beta_{2}}, \ldots, \sqrt{\beta_{n}}\right)$. It is clear that $O \leq W_{0} \leq I$. Then,

$$
A=U_{1}^{*} W_{0} U_{2} B V_{2} W_{0} V_{1}^{*} .
$$

Let $W=U_{1}^{*} W_{0} U_{2}$ and $V=V_{2} W_{0} V_{1}^{*}$. It is trivial that both $W$ and $V$ are contractive. Then, $A=W B V$.

Let $A$ and $B$ be positive semidefinite. Note that for positive semidefinite matrices, singular values and eigenvalues are the same. Using the spectral decompositions of $A$ and $B$, the unitary matrices $U_{1}$ and $U_{2}$ exist such that

$$
\begin{aligned}
& U_{1}^{*} A U_{1}=\operatorname{diag}\left(s_{1}(A), s_{2}(A), \ldots, s_{n}(A)\right), \\
& U_{2}^{*} B U_{2}=\operatorname{diag}\left(s_{1}(B), s_{2}(B), \ldots, s_{n}(B)\right) .
\end{aligned}
$$
proof.

The left proof is similar to the above. This completes the

It is clear that for $A \in M_{n}$,

$$
s_{j}(A)=s_{j}(A \oplus O), \quad j=1,2, \ldots, n,
$$

where $O$ is a zero square matrix of any order. An interesting consequence of Theorem 1 is the following.

Corollary 1 (see [4], Theorem 2.1). Let $A_{i}, B_{i}, X_{i} \in M_{n}, i=$ $1, \ldots, m$. Then

$$
s_{j}\left(\sum_{i=1}^{m} A_{i} X_{i} B_{i}\right) \leq\left\|\sum_{i=1}^{m}\left|A_{i}^{*}\right|^{2}\right\|^{1 / 2}\left\|\sum_{i=1}^{m}\left|B_{i}\right|^{2}\right\|^{1 / 2} s_{j}\left(\bigoplus_{i=1}^{m} X_{i}\right),
$$

for $j=1, \ldots, n$.
Proof. Denote

$$
\begin{aligned}
A & =\left(\begin{array}{cccc}
A_{1} & A_{2} & \cdots & A_{m} \\
0 & 0 & \cdots & 0 \\
\vdots & \vdots & \ddots & \vdots \\
0 & 0 & \cdots & 0
\end{array}\right), \\
B & =\left(\begin{array}{cccc}
B_{1} & 0 & \cdots & 0 \\
B_{2} & 0 & \cdots & 0 \\
\vdots & \vdots & \ddots & \vdots \\
B_{m} & 0 & \cdots & 0
\end{array}\right), \\
X & =\bigoplus_{i=1}^{m} X_{i} .
\end{aligned}
$$

Then,

Then, $\quad\|A\|=\left\|\sum_{i=1}^{m}\left|A_{i}^{*}\right|^{2}\right\|^{1 / 2}$ and $\|B\|=\left\|\sum_{i=1}^{m}\left|B_{i}\right|^{2}\right\|^{1 / 2}$.

$$
\frac{A}{\|A\|} X \frac{B}{\|B\|}=\frac{1}{\|A\|\|B\|}\left(\sum_{i=1}^{m} A_{i} X_{i} B_{i}\right) \oplus O,
$$

where $O$ is the zero matrix of order $n(m-1)$. It is clear that both $(1 /\|A\|) A$ and $(1 /\|B\|) B$ are contractive matrices. By Theorem 1, this completes the proof.

The following result generalizes the result due to Hirzallah and Kittaneh ([4], Corollary 2.1).

Theorem 2. Let $A_{1}, A_{2}, \ldots, A_{m} \in M_{n}$, and let $\lambda_{1}, \lambda_{2}, \ldots$, $\lambda_{m}$ be complex numbers such that $\left|\lambda_{1}\right|+\left|\lambda_{2}\right|+\cdots+\left|\lambda_{m}\right| \leq 1$. Then, for $j=1, \ldots, n$,

$$
s_{j}\left(\lambda_{1} A_{1}+\lambda_{2} A_{2}+\cdots+\lambda_{m} A_{m}\right) \leq s_{j}\left(A_{1} \oplus A_{2} \oplus \cdots \oplus A_{m}\right) .
$$

Proof. Note that each $\lambda_{k}=\left|\lambda_{k}\right| e^{i \theta_{k}}, k=1, \ldots, m$, for some $\theta_{k} \in[0,2 \pi]$. Denote

$$
\begin{aligned}
A & =\left(\begin{array}{cccc}
\sqrt{\left|\lambda_{1}\right|} I_{n} & \sqrt{\left|\lambda_{2}\right|} I_{n} & \cdots & \sqrt{\left|\lambda_{m}\right|} I_{n} \\
0 & 0 & \cdots & 0 \\
\vdots & \vdots & \ddots & \vdots \\
0 & 0 & \cdots & 0
\end{array}\right), \\
B & =\left(\begin{array}{cccc}
\sqrt{\left|\lambda_{1}\right|} e^{i \theta_{1}} I_{n} & 0 & \cdots & 0 \\
\sqrt{\left|\lambda_{2}\right|} e^{i \theta_{2}} I_{n} & 0 & \cdots & 0 \\
\vdots & \vdots & \ddots & \vdots \\
\sqrt{\left|\lambda_{m}\right|} e^{i \theta_{m}} I_{n} & 0 & \cdots & 0
\end{array}\right) \\
X & =\bigoplus_{i=1}^{m} A_{i} .
\end{aligned}
$$

Then,

$$
A X B=\left(\lambda_{1} A_{1}+\lambda_{2} A_{2}+\cdots+\lambda_{m} A_{m}\right) \oplus O_{n m-n},
$$

with contractive matrices $A$ and $B$. By Theorem 1, this completes the proof. 
Remark. This result admits the following important special cases:

(1) Let $A_{1}, A_{2}, \ldots, A_{m} \in M_{n}$, and let $\lambda_{1}, \lambda_{2}, \ldots, \lambda_{m}$ be nonnegative real numbers with $\lambda_{1}+\lambda_{2}+\cdots+$ $\lambda_{m}=1$. Then,

$$
\begin{array}{r}
s_{j}\left(\lambda_{1} A_{1}+\lambda_{2} A_{2}+\cdots+\lambda_{m} A_{m}\right) \leq s_{j}\left(A_{1} \oplus A_{2} \oplus \cdots \oplus A_{m}\right), \\
j=1, \ldots, n .
\end{array}
$$

(2) Let $A, B \in M_{n}$. Then,

$$
s_{j}\left(\frac{A+i B}{2}\right) \leq s_{j}(A \oplus B), \quad j=1, \ldots, n .
$$

In particular, letting $B=i A^{*}$, we have

$$
s_{j}(\operatorname{Im} A) \leq s_{j}(A \oplus A), \quad j=1, \ldots, n,
$$

which can be regarded as a complement to Hirzallah and Kittaneh's inequality (see ([4], Corollary 2.3)), which says

$$
s_{j}(\operatorname{Re} A) \leq s_{j}(A \oplus A), \quad j=1, \ldots, n,
$$

where $\operatorname{Re} A$ and $\operatorname{Im} A$ denote the real part and the imaginary part of $A$, respectively.

Applying Theorem 2, we have the following result.

Corollary 2. Let $A \in M_{n}$. Then,

$$
s_{j}(A) \leq s_{j}\left(\left(A+A^{*}\right) \oplus\left(A-A^{*}\right)\right), \quad j=1, \ldots, n .
$$

Proof. Considering the Cartesian decomposition of $A, A=\operatorname{Re} A+i \operatorname{Im} A$. By Theorem 2 , we have for $1 \leq j \leq n$,

$$
\begin{aligned}
s_{j}(A) & =2 s_{j}\left(\frac{\operatorname{Re} A+i \operatorname{Im} A}{2}\right) \leq 2 s_{j}(\operatorname{Re} A \oplus \operatorname{Im} A) \\
& =s_{j}\left(\left(A+A^{*}\right) \oplus\left(A-A^{*}\right)\right) .
\end{aligned}
$$

The last equality on the above is due to the fact that singular values are unitarily invariant: $s_{j}(U A V)=s_{j}(A), j=1, \ldots, n$, for every $A$ and all unitary $U$ and $V$.

Remark. Next, we show that equality is possible in inequality (20) for some nonzero square matrix $T$. Consider the matrix $T=\left(\begin{array}{ll}0 & 1 \\ 0 & 0\end{array}\right)$. Then,

$$
\begin{aligned}
& T+T^{*}=\left(\begin{array}{ll}
0 & 1 \\
1 & 0
\end{array}\right), \\
& T-T^{*}=\left(\begin{array}{cc}
0 & 1 \\
-1 & 0
\end{array}\right) .
\end{aligned}
$$

It is clear that $s_{1}(T)=s_{1}\left(\left(T+T^{*}\right) \oplus\left(T-T^{*}\right)\right)=1$.
The well-known arithmetic-geometric mean inequality for singular values due to Bhatia and Kittaneh [6] says that if $A, B \in M_{n}$, then

$$
2 s_{j}\left(A B^{*}\right) \leq s_{j}\left(A A^{*}+B B^{*}\right), \quad j=1, \ldots, n .
$$

Zhan [5] asserts that for $A, B \in M_{n}$, if $A \geq O$ and $B \geq O$, then

$$
s_{j}(A-B) \leq s_{j}(A \oplus B), \quad j=1, \ldots, n .
$$

In [7], Tao has proved that if $A, B, C \in M_{n}$ such that $\left(\begin{array}{cc}A & B \\ B^{*} & C\end{array}\right) \geq O$, then

$$
s_{j}(B) \leq \frac{1}{2} s_{j}\left(\begin{array}{cc}
A & B \\
B^{*} & C
\end{array}\right), \quad j=1, \ldots, n .
$$

It has been pointed out [7] that the three inequalities (23)-(25) are equivalent.

Recently, Audeh and Kittaneh [8] proved that let $A, B, C \in M_{n}$. If $\left(\begin{array}{cc}A & B \\ B^{*} & C\end{array}\right) \geq O$, then

$$
s_{j}(B) \leq s_{j}(A \oplus C), \quad j=1, \ldots, n .
$$

Next, we will interpolate Audeh and Kittaneh's inequality (26) by proving.

Theorem 3. Let $A, B, C \in M_{n}$ such that $\left(\begin{array}{cc}A & B \\ B^{*} & C\end{array}\right) \geq O$.
Then,

$$
s_{j}(B) \leq \frac{1}{2} s_{j}\left(\begin{array}{cc}
A & B \\
B^{*} & C
\end{array}\right) \leq s_{j}(A \oplus C), \quad j=1, \ldots, n .
$$

Proof. Since $\left(\begin{array}{cc}A & B \\ B^{*} & C\end{array}\right) \geq O$, we have

$$
\left(\begin{array}{ll}
A & B \\
B^{*} & C
\end{array}\right)=\left(\begin{array}{ll}
M & N
\end{array}\right)^{*}\left(\begin{array}{ll}
M & N
\end{array}\right)
$$

for some $M, N \in M_{2 n, n}$. Then,

$$
\begin{aligned}
& A=M^{*} M, \\
& C=N^{*} N .
\end{aligned}
$$

Note that singular values and eigenvalues of positive semidefinite matrices are the same. By Theorem 1.27 in [3], for $1 \leq j \leq n$, then

$$
\begin{aligned}
s_{j}\left(\begin{array}{cc}
A & B \\
B^{*} & C
\end{array}\right) & =s_{j}\left(\left(\begin{array}{ll}
M & N
\end{array}\right)^{*}\left(\begin{array}{ll}
M & N
\end{array}\right)\right) \\
& =s_{j}\left(\left(\begin{array}{ll}
M & N
\end{array}\right)\left(\begin{array}{ll}
M & N
\end{array}\right)^{*}\right) .
\end{aligned}
$$

Appling Theorem 2 and Theorem 1.27 in [3], we obtain that for $1 \leq j \leq n$,

$$
s_{j}\left(M M^{*}+N N^{*}\right) \leq 2 s_{j}\left(M M^{*} \oplus N N^{*}\right)=2 s_{j}\left(M^{*} M \oplus N^{*} N\right),
$$


i.e.,

$$
\frac{1}{2} s_{j}\left(\begin{array}{cc}
A & B \\
B^{*} & C
\end{array}\right) \leq s_{j}(A \oplus C) .
$$

Combining inequality (25) by Tao and the above inequality gives (27).

Let $A=U \operatorname{diag}\left(\lambda_{1}, \lambda_{2}, \ldots, \lambda_{n}\right) U^{*}$ be the spectral decomposition of $A \in H_{n}$ with $U$ unitary and $\lambda_{1} \geq \cdots \geq \lambda_{p}$ $\geq 0>\lambda_{p+1} \geq \cdots \geq \lambda_{n}$. Denote

$$
\begin{aligned}
& A^{+}=U \operatorname{diag}\left(\lambda_{1}, \ldots, \lambda_{p}, 0, \ldots, 0\right) U^{*}, \\
& A^{-}=U \operatorname{diag}\left(0, \ldots, 0,-\lambda_{p+1}, \ldots,-\lambda_{n}\right) U^{*} .
\end{aligned}
$$

Then $A^{+} \geq O, A^{-} \geq O$, and $A=A^{+}-A^{-}$. This is called the Jordan decomposition of $A$. As is known, there are at least four different proofs of Zhan's result [5, 9]. Applying Theorem 1 and the Jordan decomposition, we give another direct proof of Zhan's inequality (8).

Proof. Denote $T=A-B \in H_{n}$. Considering the Jordan decomposition of $T, T=T^{+}-T^{-}$. Recall from ([3], Theorem 3.6) that if $T=A-B$ with $A \geq O, B \geq O$, then

$$
\begin{aligned}
s_{j}\left(T^{+}\right) \leq s_{j}(A), & \\
s_{j}\left(T^{-}\right) \leq s_{j}(B), & \\
& j=1, \ldots, n .
\end{aligned}
$$

By Theorem 1, there exist two contractive matrices $W_{1}$ and $W_{2}$ such that

$$
\begin{aligned}
& T^{+}=W_{1}^{*} A W_{1}, \\
& T^{-}=W_{2}^{*} A W_{2} .
\end{aligned}
$$

Denote $W=W_{1} \oplus W_{2}$. Then, $W$ is a contractive matrix and so $T^{+} \oplus T^{-}=W^{*}(A \oplus B) W$. For $T \in H_{n}$,

$$
s_{j}(T)=s_{j}(T \oplus O)=s_{j}\left(T^{+} \oplus T^{-}\right), \quad j=1, \ldots, n,
$$

where $O \in M_{n}$. By Theorem 1, this completes the proof. [10].

\section{Data Availability}

No data were used to support this study.

\section{Conflicts of Interest}

The authors declare there are no conflicts of interest.

\section{Authors' Contributions}

The authors read and approved the final manuscript.

\section{Acknowledgments}

This work was supported by the Anhui Provincial Natural Science Foundation (1708085QA05) and the Key Program in the Youth Elite Support Plan in Universities of Anhui Province (gxyqZD2018047).

\section{References}

[1] R. Bhatia, Matrix Analysis, Springer-Verlag, Berlin, Germany, 1997.

[2] X. Zhan, "Matrix inequalities," Lecture Notes in Mathematics, Vol. 1790, Springer-Verlag, Berlin, Germany, 2002.

[3] X. Zhan, "Matrix Theory," Graduate Studies in Mathematics, Vol. 147, American Mathematical Society, Providence, RI, USA, 2013.

[4] O. Hirzallah and F. Kittaneh, "Inequalities for sums and direct sums of Hilbert space operators," Linear Algebra and its Applications, vol. 424, no. 1, pp. 71-82, 2007.

[5] X. Zhan, "Singular values of differences of positive semidefinite matrices," SIAM Journal on Matrix Analysis and Applications, vol. 22, no. 3, pp. 819-823, 2001.

[6] R. Bhatia and F. Kittaneh, "On the singular values of a product of operators," SIAM Journal on Matrix Analysis and Applications, vol. 11, no. 2, pp. 272-277, 1990.

[7] Y. Tao, "More results on singular value inequalities of matrices," Linear Algebra and Its Applications, vol. 416, no. 2-3, pp. 724-729, 2006.

[8] W. Audeh and F. Kittaneh, "Singular value inequalities for compact operators," Linear Algebra and its Applications, vol. 437, no. 10, pp. 2516-2522, 2012.

[9] X. Zhan, "On some matrix inequalities," Linear Algebra and its Applications, vol. 376, pp. 299-303, 2004. 